\title{
Effects of Pediatric Emergence Delirium Education on Analgesic Administration by PACU Nurses: A Pilot Study
}

\author{
Melinda Nwanganga ${ }^{1}$, Jean Cadet $^{2} \&$ Jochebed Ade-Oshifogun ${ }^{1}$ \\ ${ }^{1}$ School of Nursing, Andrews University, Berrien Springs, MI, USA \\ ${ }^{2}$ School of Public Health, Nutrition and Wellness, Andrews University, Berrien Springs, MI, USA \\ Correspondence: Samuel P. Abraham, Associate Professor of Nursing, Bethel University, 1001 Bethel Circle, \\ Mishawaka, Indiana, USA.
}

Received: February 10, 2020

Accepted: February 28, 2020

Online Published: March 9, 2020

doi:10.20849/ijsn.v5i1.720

URL: https://doi.org/10.20849/ijsn.v5i1.720

\begin{abstract}
Background: Emergence delirium (ED) is a behavioral disturbance as a result of general anesthesia that commonly occurs in pediatric patients. Adverse effects of ED lead to a complicated recovery from anesthesia leading to the increased use of sedatives and analgesics. Due to the multiple ramifications of ED, PACU nurses need to provide appropriate care to promote a safe recovery process from anesthesia while avoiding unnecessary use of analgesics. The Pediatric Assessment Emergence Delirium (PAED) tool has been successfully utilized in recognizing ED in PACU. Purpose: The purpose of this evidence-based practice project was to educate PACU nurses about the use of the PAED tool, and explore the post-education effect on the nurses' use of analgesics in the immediate post-op period. Method: This project utilized a pre- and post- interventional study design. Twenty-eight charts of pediatric post-op patients were screened pre-intervention for analgesic use. An educational session on ED and instruction on the use of the PAED tool were provided to PACU nurses. After six weeks, 24 charts were reviewed for analgesic administration. The results of pre and post analgesic use were compared by using Fisher's exact test of independence. Results: Findings revealed an overall non-significant decrease in analgesic use from $21 \%(\mathrm{n}=6)$ to $17 \%(\mathrm{n}=4)$ between pre-intervention and post-intervention groups $\left(x^{2}=0.189, p=.47\right)$, though a decrease in the use of stadol was observed from $11 \%(n=3)$ to $0 \%$. Conclusion: ED education with PAED use has the potential to influence PACU nurses' usage of analgesics. Follow up studies with larger sample sizes are needed to explore such an influence further.
\end{abstract}

Keywords: analgesia, emergence agitation, emergence delirium, pacu, pediatric, post-anesthesia, recovery

\section{Introduction and Background}

Emergence delirium (ED) has been established as a common occurrence after general anesthesia. First described by Eckenhoff, Kneale, \& Dripps (1961), ED, also called emergence agitation, is a post-anesthetic phenomenon. Although it can affect all populations receiving anesthesia, it is more prevalent in the pediatric population. Its manifestation among pediatric patients is described as, "a dissociated state of consciousness in which the child is inconsolable, irritable, uncompromising or uncooperative, typically thrashing, crying, moaning, and incoherent," (Hudek, 2009, p.510). ED is a transient condition occurring in the immediate postoperative period when the patient is emerging from general anesthesia. Although ED is typically self-limiting and generally resolves within 30 minutes, it is associated with several complications, adversely affecting the recovery process for pediatric surgical patients. PACU nurses must be familiar with ED and knowledgeable in managing potential complications during the recovery process for this particular population. PACU nurses are in the position of managing ED directly being the primary caregivers during the immediate postoperative phase. Being able to identify and understand this phenomenon readily will direct the clinical decisions that nurses make regarding how to treat it best.

The clinical setting in which implementation of this project took place has been established as the region's only comprehensive children's hospital. The facility offers a wide variety of surgical treatments for pediatric patients in the local area, as well as those referred from more than 20 surrounding hospitals in northern Indiana and southwest Michigan. The organization has the overall goal of improving the quality of care provided to pediatric patients, making it an ideal setting for this evidence-based practice (EBP) project, which aims to improve the management of ED in pediatric surgical patients. 
The PACU nurses in the clinical agency, at the time of this project, were not trained in the recognition or management of ED. Anesthesiologists provide a blanket of post-operative medication orders to be given on an as-needed basis for the control of nausea and pain while in the PACU. Nurses were expected to use their clinical judgment to decide how to manage the patient's recovery process. When a child wakes up agitated, the cause of the agitation must be identified for appropriate treatment to occur. The origins of agitation may include anxiety, pain, and delirium among others and may be challenging to differentiate between the various causes (Voepel-Lewis, Burke, Hadden, Tait, \& Malviya, 2005).

Consistent among the literature about ED is the multifactorial risks associated with its occurrence as well as the observed behavior. Per evidence in the literature, best practice recommendations include accurately identifying the occurrence of ED (Mohkamkar, Farhoudi, Alam-Sahebpour, Mousavi, Khani, \& Shahmohammadi, 2014; Stamper, Hawks, Taicher, Bonta, \& Brandon, 2014). Some characteristics of ED are comparable to postoperative pain and while this can be a confounding factor of ED, it is still necessary to differentiate between the two.

Various assessment scales and tools have been used to identify ED. However, many involve the rating of behavior that is not specific to ED. The Cravero scale has five categories ranging from obtunded and unresponsive to wild thrashing behavior requiring restraint. A score of 4 (from crying and difficult to console to wild thrashing) for a five or more-minute duration despite active calming efforts is regarded as indicative of ED (Reduque \& Verghese, 2013). The Watcha scale is another simple scale with scoring ranging from asleep to agitated and thrashing around (Reduque \&Verghese, 2013). Patients with scores greater than or equal to three were identified in this scale as experiencing emergence delirium. In identifying pain among pediatric patients, the Faces, Legs, Activity, Cry, and Consolability (FLACC) scale is one of the most widely utilized observational pain assessment scales in clinical practice. The tool was designed and validated to assess postoperative pain in patients who are unable to verbalize pain symptoms. The accurate assessment leads to improved pain management in this patient population. However, among the tools developed to measure ED, the PAED scale is the most widely used and validated means of identifying delirium (Mason, 2017). Sikich and Lerman (2004) developed the PAED tool which has provided evidence of being a more sensitive tool in measuring ED in pediatric surgical patients than other tools used. The PAED tool offers definite proof of measurement reliability and validity (Stamper et al., 2014). This tool uses a theoretical framework of delirium, focusing on changes in consciousness and cognition, which permits differentiation of emergence delirium behaviors from those behaviors associated with pain (Stamper et al., 2014). In several observational studies, the implementation of the PAED Scale was evaluated for effectiveness in identifying ED (Bong \& Ng, 2009; Bortone et al., 2014; Mohkamkar et al., 2014; Stamper et al., 2014).

Consequently, caring for the agitated child requires a thorough assessment and targeted nursing interventions. PACU nurses should have the knowledge to correctly identifying ED so they can make informed clinical decisions regarding the care of the pediatric surgical patient in the immediate post-operative period. The purpose of this evidence-based practice project was to educate PACU nurses about ED and the use of the PAED tool. The post-education effect on nurses' use of analgesics in the immediate post-op period was also explored.

\section{Methodology}

\subsection{Participants}

The participants of this EBP project consisted of registered nurses in the PACU department who rotate to the outpatient surgical care unit. There are 33 nurses in the post-anesthesia department. Eighteen of these nurses work in the outpatient surgery center (OSC) and regularly recover pediatric surgical patients emerging from general anesthesia. The project manager obtained approval from the clinical agency's internal review board (IRB) and Valparaiso University's IRB for the implementation of the project. Consent was implied by nurses' attendance of the education sessions.

\subsection{Instrument}

The PAED scale (Sikich \& Lerman, 2004) was utilized in this project to measure the incidence of ED among pediatric surgical patients recovering in the PACU. It is a five-component rating scale based on a nurse's evaluation of a child's behavior. Components include: "the child makes eye contact with the caregiver," "the child's actions are purposeful," "the child is aware of his/her surroundings," "the child is restless," and "the child is inconsolable." The first three components are reversed scored as follows: $4=$ not at all, $3=$ just a little, 2 = quite a bit, $1=$ very much, $0=$ extremely. While the fourth and fifth items on the scale are scored as follows: $0=$ not at all, $1=$ just a little, $2=$ quite a bit, $3=$ very much, $4=$ extremely. The scores were then summed to obtain a total PAED score. A PAED score of 10 or greater identifies the presence of ED. The severity of ED increases directly with the PAED score. The internal consistency of the PAED scale was .89 and the reliability 
was .84 with a confidence interval of $95 \%$ (Stamper et al., 2014). The Cronbach's alpha for the PAED tool with this project was .90 demonstrating a high internal consistency.

\subsection{Data Collection Method}

Data was collected through chart reviews. All pediatric surgical patients treated during the 12 weeks of the project implementation were reviewed for inclusion in the project. Inclusion criteria included ages 1- 18, patients undergoing outpatient surgical procedures, and patients undergoing general anesthesia. A baseline data set of 28 patients' records met the inclusion criteria. Information extracted from these charts included age, length of stay, race, weight, pre-medication, type of surgery, type of anesthesia, preoperative and intraoperative medications administered by anesthesiologists, and postoperative analgesics administered by PACU nurses. The above data was collected before and after the educational intervention. Twenty-four records met the inclusion criteria after the intervention.

\subsection{Intervention}

The education presentation provided to PACU nurses included the definition of ED, associated complications, risk factors, and a summary of management methods. The presentation was completed during an in-service staff program utilizing discussion, PowerPoint Presentation, and handouts. The presentation consisted of five 30 minutes sessions over three days. A total of 14 nurses attended the educational presentation while the remaining four were given a handout and an electronic copy of the PowerPoint presentation via email. Nurses were taught how to use Sikich and Lerman's (2004) PAED scale and were given a laminated, pocket-sized card featuring the scale. After the presentation, PACU nurses had the opportunity to assess a case study scenario to demonstrate proper utilization of the PAED assessment tool, practice utilization of the scale on patients and ask any further questions regarding ED. Nurses were to assess and record PAED scores on all pediatric patients recovering from general anesthesia in the OSC PACU department post-presentation. The project manager observed the nurse participants for two days post-presentation to ensure proper utilization of the tool.

\subsection{Variables}

The main variables for this project were the total number of times analgesics were used, the type of analgesic used, and the use of the PAED tool. Standard analgesics administered and therefore, measured in this facility include Fentanyl, Morphine, Stadol, and Tylenol.

\subsection{Data Analysis}

The data was analyzed using SPSS (IBM SPSS Statistics for Windows, version 25). Descriptive statistics were completed for the demographic data. Because of the small sample sizes, Fisher's exact test of independence was used to compare analgesics usage between pre and post-test data and to determine if there is any relationship between the use of analgesics in pediatric post-op patients and the patients' ED score using the PAED tool. Alpha $(\alpha)$ was set at .05 . The ED score variable was utilized for grouping. Patients with scores $<12=$ lower score group, and score $\geq 12$ higher score group.

\section{Results}

\subsection{Demographic Data}

Table 1 below showed a summary of the demographic data. The pre and post-intervention groups did not differ significantly in demographics, though there are differences in the percentages of patients having different types of surgical intervention. 
Table 1

Mdn, (maximum, minimum), and percentages for patients' demographics

\begin{tabular}{|c|c|c|c|c|}
\hline \multirow[b]{2}{*}{ Characteristics } & \multicolumn{2}{|c|}{$\begin{array}{l}\text { Group 1 } \\
(\mathrm{N}=28)\end{array}$} & \multicolumn{2}{|c|}{$\begin{array}{l}\text { Group } 2 \\
(\mathrm{~N}=24)\end{array}$} \\
\hline & $n$ & $\%$ & $n$ & $\%$ \\
\hline Age in years & & $6(5,8)$ & & $5(1,18)$ \\
\hline Weight & & $20(15,37)$ & & $20(9,88)$ \\
\hline Length of stay & & $26(14,85)$ & & $28(11,85)$ \\
\hline \multicolumn{5}{|l|}{ Race } \\
\hline White & 18 & $64 \%$ & 14 & $58 \%$ \\
\hline Black & 6 & $21 \%$ & 3 & $13 \%$ \\
\hline Hispanic & 2 & $7 \%$ & 5 & $21 \%$ \\
\hline Asian & 1 & $4 \%$ & & \\
\hline Other & 1 & $4 \%$ & 2 & $8 \%$ \\
\hline \multicolumn{5}{|l|}{ Type of surgery } \\
\hline Dental & 19 & $68 \%$ & 8 & $33 \%$ \\
\hline Orthopedics & 1 & $4 \%$ & 1 & $4 \%$ \\
\hline Urology & 2 & $7 \%$ & 1 & $4 \%$ \\
\hline Eye, Nose, Throat & 3 & $11 \%$ & 11 & $46 \%$ \\
\hline General procedures* & 3 & $11 \%$ & 3 & $13 \%$ \\
\hline Post-op analgesics usage & 6 & $21 \%$ & 5 & $17 \%$ \\
\hline
\end{tabular}

The mean PAED score for the 24 patients that were assessed for ED after the implementation of the project was 9.67 (SD = 5.97). Eleven of them were found to have a PAED score equal to or above 12 indicating a high degree of ED. The incidence of ED at this facility during this project was $46 \%$.

Pre and Post Intervention Analgesics Use

Table 2 showed the rates of analgesic administration by PACU nurses. The overall rate declined from $21 \%(\mathrm{n}=6)$ to $17 \%(\mathrm{n}=4)$ between pre-intervention and post-intervention groups, but the decline was not significant $\left(\chi^{2}=\right.$ $0.189, \mathrm{p}=.47)$.

Table 2

The proportion of pre and post intervention analgesics use among all pediatrics patients

\begin{tabular}{llllll}
\hline & Fentanyl & Morphine & Stadol & Tylenol & Total* \\
\hline Pre $(\mathrm{N}=28)$ & $4 \%(\mathrm{n}=1)$ & $7 \%(\mathrm{n}=2)$ & $11 \%(\mathrm{n}=3)$ & $0 \%(\mathrm{n}=0)$ & $21 \%(\mathrm{n}=6)$ \\
\hline Post $(\mathrm{N}=24)$ & $13 \%(\mathrm{n}=3)$ & $4 \%(\mathrm{n}=1)$ & $0 \%(\mathrm{n}=0)$ & $4 \%(\mathrm{n}=1)$ & $17 \%\left(\mathrm{n}=5^{*}\right)$ \\
\hline Test & $\begin{array}{l}\chi^{2}=1.451 \\
p=.249\end{array}$ & $\begin{array}{l}\chi^{2}=0.211 \\
p=.559\end{array}$ & & $\begin{array}{l}\chi^{2}=0.189 p \\
=.47\end{array}$ \\
\hline
\end{tabular}

* One person received both fentanyl and morphine 
The frequency of analgesics uses decreased among all analgesics with the exception of fentanyl and Tylenol, which both increased. The rate at which fentanyl and acetaminophen were administered increased post-intervention, though those differences were not significant (See Table 2).

\subsection{PAED Scores and Analgesics Use}

Patients that had lower ED scores $(<12)$ from the PAED tools tended to not receive as many analgesics $(15 \%)$ compared to patients with higher ED scores (18\%), but that difference was not significant. (see Table 3 below). This reassured us that the nurses understood the use of the PAED tool and are making correct clinical decisions based on the instrument.

Table 3

PAED score vs. use of analgesics

\begin{tabular}{|c|c|c|}
\hline Outcome & $\begin{array}{l}\text { Use of Analgesics } \\
(\mathrm{n}=11)\end{array}$ & $\begin{array}{l}\text { Nonuse of analgesics } \\
(\mathrm{n}=13)\end{array}$ \\
\hline Lower ED Score & $15 \%$ & $85 \%$ \\
\hline$(n=13)$ & $(n=2)$ & $(\mathrm{n}=11)$ \\
\hline Higher ED score & $18 \%$ & $82 \%$ \\
\hline$(\mathrm{n}=11)$ & $(\mathrm{n}=2)$ & $(\mathrm{n}=9)$ \\
\hline$\chi^{2}=0.034, p=.63$ & & \\
\hline
\end{tabular}

\section{Discussion}

According to the literature, ED ranges from $25-80 \%$, depending on the definition used to measure it (Sikich \& Lerman, 2004). In a study by Stamper et al. (2014), the incidence of ED was identified more often using the PAED scale than another LOC-RASS scale. This project utilized the PAED scale and determined the incidence of ED at $46 \%$, consistent with reported rates of ED in the literature (Liang, Zhou, Ni, Luo, \& Liu, 2014; Somaini, Engelhardt, Fumagalli, \& Ingelmo, 2016828).

Several studies support the use of analgesics such as fentanyl, to be used for the treatment of ED (Bortone et al, 2014; Liang, Zhou, Ni, Luo, \& Liu, 2014; Voepel-Lewis et al., 2005). In this project, the use of fentanyl and acetaminophen increased even though not significantly. Among the literature, fentanyl was repeatedly found to be an effective treatment for ED (Bortone et al, 2014; Liang et al., 2014; Voepel-Lewis et al., 2005). This treatment option was discussed in the educational intervention and this may explain why the increase in fentanyl administration, although the increase was not statistically significant. Furthermore, a possible explanation for the increase in acetaminophen administration could be that nurses were more aware of the possibility of overmedicating and chose a less potent analgesic to manage pain to avoid administering unnecessary narcotics. Another point highlighted in the educational intervention was the potential for mistaking manifestations of ED for pain and the consequent inappropriate treatment. This may explain the significant decrease in the use of stadol since this medication is a narcotic pain reliever and was not recommended in the literature.

A possible barrier to implementing the use of the assessment tool into standard care was the resistance to change current practice among nurses in this facility. The majority (over 75\%) of nurses had more than five years of experience in the PACU and over 15 years of general nursing experience. The high level of experienced nurses involved in this project may have affected how readily they would accept a new approach to care. While clinical decision-making skills are gained from education and training, it is also heavily influenced by experience. Experienced nurses may rely more on previous experiences to rule out problems rather than using new tools with no experience. This project was presented to the nurses as a pilot project as opposed to a change in policy. This also may have been a barrier for nurses. Although encouraged by the clinical site facilitator, it was not mandatory and therefore may have been perceived as optional for the nursing staff to complete.

\subsection{Limitations and Future Projects}

Limitations in the project may include the lack of interaction between administrative leaders and the nurses regarding this project. Although the administrative leaders were supported and involved in the planning of the project, they were less involved and less visible during its implementation. This may have affected the nurses' views of the importance of the change itself and their role in the change. Another limitation of the project involved the electronic record. The PAED tool was not included in the electronic health record. Therefore, the nurses possibly viewed the tool as additional work. Having the PAED scale listed among nursing assessments 
would have improved compliance with its use. Small sample size and time frame were other limitations to the study. A larger sample size may result in significant findings. Given more time and opportunities to incorporate the training into clinical practice may also have resulted in more positive outcomes.

\subsection{Implications for Further Study}

Among the causes of ED in the literature, preoperative anxiety was prevalent in the development of ED. Further research is needed for a more preventative approach to nursing care for patients at risk of developing ED. Preventative care must include a holistic approach that encompasses influences such as the child's family, prior experience, and environmental factors. Further study on the nurse's role in addressing preoperative anxiety, and consequently preventing ED, is needed to continue the progress in caring for this select population group.

\section{Conclusion}

The development of ED in the immediate post-operative period continues to be a common occurrence among pediatric surgical patients emerging from general anesthesia. The findings of this project demonstrate that ED education may influence PACU nurses' usage of analgesics. PACU nurses' knowledge of the phenomenon, use of a valid and reliable assessment tool, and effective treatment options may help to provide appropriate care to this vulnerable population group.

\section{References}

Bong, C. L., Ng, A. S. B. (2009). Evaluation of emergence delirium in Asian children using the pediatric anesthesia emergence delirium scale. Pediatric Anesthesia, 19, 593-600. https://doi.org/10.1111/j.1460-9592.2009.03024.x

Bortone, L., Bertolizio, G., Engelhardt, T., Frawley, G., Somaini, M., \& Ingelmo, P. (2014). The effect of fentanyl and clonidine on early postoperative negative behavior in children: A double-blind placebo-controlled trial. Pediatric Anesthesia, 24, 614-619. https://doi.org/10.1111/pan.12388

Eckenhoff, J. E., Kneale, D. H., \& Dripps, R. D. (1961). The incidence and etiology of postanesthetic excitement: A clinical survey. Anesthesiology, 9(22), 667-673.

Hudek, K. (2009). Emergence delirium: A nursing perspective. AORN Journal, 89(3), 509-520. https://doi.org/10.1016/j.aorn.2008.12.026

Liang, P., Zhou, C., Ni, J., Luo, Z., \& Liu, B. (2014). Single-dose sufentanil or fentanyl reduces agitation after sevoflurane anesthesia in children undergoing ophthalmic surgery. Pakistan Journal of Medical Sciences, 30(5), 1059-1063. https://doi.org/10.12669/pjms.305.4483

Mason, K. P. (2017). Paediatric emergence delirium: A comprehensive review and interpretation of the literature. BJA: British Journal of Anaesthesia, 118(3), 335-343. https://doi.org/10.1093/bja/aew477

Mohkamkar, M., Farhoudi, F., Alam-Sahebpour, A., Mousavi, S., Khani, S., \& Shahmohammadi, S. (2014). Postanesthetic emergence agitation in pediatric patients under general anesthesia. Iranian Journal of Pediatrics, 24(2), 184-190.

Reduque, L. L., \& Verghese, S. T. (2013). Paediatric emergence delirium. Continuing Education in Anaesthesia Critical Care \& Pain, 13(2), 39-41.

Sikich, N., \& Lerman, J. (2004). Development and psychometric evaluation of the pediatric anesthesia $\begin{array}{llll}\text { emergence delirium } & \text { scale. } & \text { Anesthesiology, } & \text { 100(5), }\end{array}$ https://doi.org/10.1097/00000542-200405000-00015

Somaini, M., Engelhardt, T., Fumagalli, R., \& Ingelmo, P. M. (2016). Emergence delirium or pain after anaesthesia--how to distinguish between the two in young children: A retrospective analysis of observational studies. British Journal of Anaesthesia, 116(3), 377-383. https://doi.org/10.1093/bja/aev5520

Stamper, M. J., Hawks, S. J., Taicher, B. M., Bonta, J., \& Brandon, D. H. (2014). Identifying pediatric emergence delirium by using the PAED scale: A quality improvement project. AORN Journal, 99(4), 480-494. https://doi.org/10.1016/j.aorn.2013.08.019

Voepel-Lewis, T., Burke, C., Hadden, S., Tait, A., \& Malviya, S. (2005). Nurses' diagnoses and treatment decisions regarding care of the agitated child. Journal of Perianesthesia Nursing, 20(4), 239-248 https://doi.org/10.1016/j.jopan.2005.05.006

\section{Copyrights}

Copyright for this article is retained by the author(s), with first publication rights granted to the journal.

This is an open-access article distributed under the terms and conditions of the Creative Commons Attribution license (http://creativecommons.org/licenses/by/4.0/). 